\title{
Proteomic test for anti-PD-1 checkpoint blockade treatment of metastatic melanoma with and without BRAF mutations
}

Paolo A. Ascierto ${ }^{1}$, Mariaelena Capone ${ }^{1}$, Antonio M. Grimaldi', Domenico Mallardo', Ester Simeone', Gabriele Madonna ${ }^{1,2}$, Heinrich Roder ${ }^{3}$, Krista Meyer $^{3}$, Senait Asmellash ${ }^{3}$, Carlos Oliveira ${ }^{3}$, Joanna Roder ${ }^{3}$ and Julia Grigorieva ${ }^{3^{*}}$ (D)

\begin{abstract}
The therapeutic landscape in metastatic melanoma has changed dramatically in the last decade, with the success of immune checkpoint inhibitors resulting in durable responses for a large number of patients. For patients with BRAF mutations, combinations of BRAF and MEK inhibitors demonstrated response rates and benefit comparable to those from immune checkpoint inhibitors, providing the rationale for sequential treatment with targeted and immunotherapies and raising the question of optimal treatment sequencing.

Biomarkers for the selection of anti-PD-1 therapy in BRAF wild type (BRAF WT) and in BRAF mutated (BRAF MUT) patients help development of alternative treatments for patients unlikely to benefit, and might lead to better understanding of the interaction of checkpoint inhibition and targeted therapy. In this paper we evaluate the performance of a previously developed serum proteomic test, BDX008, in metastatic melanoma patients treated with anti-PD-1 agents and investigate the role of BRAF mutation status. BDX008, a pre-treatment proteomic test associated with acute phase reactants, wound healing and complement activation, stratifies patients into two groups, BDX008+ and BDX008-, with better and worse outcomes on immunotherapy.

Serum samples were available from 71 patients treated with anti-PD1 inhibitors; 25 patients had BRAF mutations, 39 were wild type. Overall, BDX008+ patients had significantly better overall survival (OS) $(H R=0.50, P=0.016)$ and a trend for better progression-free survival (PFS) $(H R=0.61, P=0.060)$ than BDX008- patients. BDX008 classification was statistically significant in the analyses adjusted for mutation status, $\mathrm{LDH}$, and line of treatment $(P=0.009$ for OS and 0.031 for PFS). BRAF WT BDX008+ patients had markedly long median OS of 32.5 months and $53 \%$ landmark 2 years survival, with statistically significantly superior OS as compared to BDX008- patients ( $H R=0.41, P=0.032)$. The difference between BDX008+ and BDX008- in PFS in BRAF WT patients and in OS and PFS in BRAF MUT patients did not reach statistical significance, though numerically was consistent with overall results. The test demonstrated significant interaction with neutrophil-to-lymphocyte ratio (NLR) (PFS $P=0.041$, OS $P=0.004$ ). BDX008 as a biomarker selecting for benefit from immune checkpoint blockade, especially in patients with wild type BRAF and in subgroups with low NLR, warrants further evaluation.
\end{abstract}

Keywords: Immunotherapy, Proteomic test, Melanoma, BRAF mutations, Anti-PD-1, Nivolumab, Pembrolizumab

\footnotetext{
*Correspondence: julia.grigorieva@biodesix.com

${ }^{3}$ Biodesix Inc, Boulder, CO, USA

Full list of author information is available at the end of the article
} 


\section{Background}

Remarkable progress in treatment of metastatic melanoma patients in the past decade led from only marginal survival benefit from chemotherapy, which was a standard of care before 2011 [1], to $20-30 \%$ of durable responses and approximately $42-47 \% 3$ years survival in advanced patients harboring $B R A F$ mutations treated with targeted therapy [2, 3]. In unselected patients treated with immune checkpoint inhibitors landmark 4 years survival was $46-53 \%[4,5]$ with durable antitumor immunity persistent 2 years after the cessation of treatment [6].

Approximately $40-50 \%$ of patients with metastatic cutaneous melanoma harbor BRAF V600 mutations, which constitutively activate the mitogen-activated protein kinase (MAPK) pathway. The BRAF inhibitors vemurafenib and dabrafenib had shown high response rates in this group of patients. Addition of downstream MEK inhibitors, such as trametinib or cobimetinib, to BRAF inhibitors, resulted in improvements in efficacy over monotherapy, with median PFS of approximately 12 months and around 20\% of patients remaining progression-free for 3 years. The new combination of encorafenib and binimetinib resulted in improved median PFS (15 months) and OS (34 months) and 3 years progression-free survival in $28 \%$ of patients [3].

Monoclonal antibodies against cytotoxic T-lymphocyte antigen-4 (CTLA-4) and programmed cell death protein 1 (PD-1) and its ligand (PD-L1) have demonstrated high activity in melanoma and other solid tumors. Ipilimumab was the first FDA-approved anti-CTLA-4 agent to achieve superiority against dacarbazine and $20 \%$ survival at 3 years and up to 10 years [7, 8]. Anti-PD-1 antibodies demonstrated good clinical activity with less toxicity than chemotherapy or ipilimumab $[9,10]$. The combination of nivolumab and ipilimumab has shown superior activity over monotherapy with either nivolumab or ipilimumab in previously untreated patients, independent of $B R A F$ status [4], however at the cost of more grade 3 or 4 adverse events.

Advanced melanoma $B R A F$ MUT patients receiving the newest combinations of BRAF and MEK inhibitors achieved outcomes similar to those of unselected patients on ipilimumab/nivolumab therapy [11]; however, immunotherapy resulted in more patients remaining progression-free in the long term. While in the BRAF WT population immunotherapy has become a standard of care, an optimal strategy in patients with $B R A F$ mutations is not that clear. Preclinical evidence suggested a synergistic effect from a combination of targeted and immunotherapies due to activation of the immune system by BRAF/MEK inhibitors and showed promising efficacy in clinical settings [12], however was hindered by a high toxicity rate [13]. Sequential treatment with immune checkpoint and BRAF/MEK inhibitors is considered more suitable for broad clinical practice; several ongoing prospective clinical trials are comparing different sequential approaches (NCT02631447, NCT02224781). It was proposed that tumors innately resistant to anti-PD-1 therapy share a transcriptional signature with melanoma cells treated with MAPK inhibitors [14]; however, it is not yet known whether a shared phenotype predicting sensitivity to BRAF inhibitors and anti-PD-1 agents exists. It would be ideal to find a test identifying either responders to targeted therapy who are unlikely to benefit more from immunotherapy or patients who may be good candidates for more aggressive treatment, such as triplet combination in patients with BRAF mutations [12]. Since BRAF and MEK inhibitors have a modifying effect on the tumor microenvironment [15], testing should be done before each new therapy type, and it is important to validate that a particular molecular test is applicable to patients with different treatment histories. It would also be advantageous to have a test that does not rely on tissue availability and could be repeated in the course of multiple lines of treatment.

The BDX008 test was developed to identify patients with better or worse outcomes when treated with immune therapies, using a cohort of ipilimumab-naïve and ipilimumab-pretreated patients from the NCT01176461 clinical study [16, 17]. The test uses matrix-assisted laser/desorption ionization (MALDI) mass spectrometry to measure the circulating proteome in blood; it requires a minimal amount of pre-treatment serum $(<10 \mu \mathrm{L})$. BDX008 has been previously validated in several independent cohorts in melanoma and lung cancers $[17,18]$.

Given the potential clinical utility of BDX008 for optimization of advanced melanoma treatment, we sought to further validate the test in an independent cohort of patients with known BRAF mutation status treated with anti-PD-1 therapy in an unselected population previously treated with ipilimumab. Considering that the majority of $B R A F$ MUT patients had received BRAF and/or MEK inhibitors in prior lines, we were interested to see whether the performance of the test would be different in this subgroup of melanoma patients. In additional exploratory analysis we have evaluated the effect of the BDX008 test depending on neutrophil-to-lymphocyte ratio (NLR), which is a surrogate marker of systemic inflammation [19] and is known to be prognostic for outcomes in melanoma and other solid tumors [20].

\section{Methods}

\section{Patients and samples}

In this retrospective observational study, 71 pre-treatment serum samples from patients receiving anti-PD-1 therapy were available for analysis and passed quality control in the generation of mass spectra.

Patients were treated with nivolumab at $3 \mathrm{mg} / \mathrm{kg}$ every 2 weeks or pembrolizumab at $2 \mathrm{mg} / \mathrm{kg}$ every 3 weeks until 
progression or occurrence of toxicity. One patient had received no prior treatment; the rest of the patients were pre-treated with ipilimumab at $3 \mathrm{mg} / \mathrm{kg}$ every 3 weeks for 4 cycles. $24 \%$ of patients were treated with immune checkpoint inhibitors in 2nd line, $75 \%$ in 3rd or higher line. 55\% of patients were $B R A F \mathrm{WT}, 35 \%$ were $B R A F$ MUT, and for $10 \%$ the $B R A F$ status was not available. $88 \%$ of $B R A F$ MUT patients had received prior treatment with vemurafenib (960 mg b.i.d.) and/or cobimetinib (60 mg/die for 21 days every 4 weeks).

Patient characteristics are summarized in Additional file 1: Table S1. Patient characteristics for the whole cohort; individual clinical data and outcomes are described in Additional file 2: Clinical information and outcomes.

\section{Spectra acquisition and processing}

Samples were processed in the same way as for the development of BDX008 using standardized operating procedures described in detail in the Supplementary materials (Additional file 3).

\section{BDX008 test}

BDX008 was previously developed using modern machine learning techniques, optimized to minimize potential for overfitting and maximize generalization to unseen data sets in cases when there are more attributes measured than samples available. One hundred nineteen samples from patients with unresectable melanoma treated with nivolumab in the scope of the NCT01176461 clinical trial were used in test development [17] (details can be found in the Supplementary materials, Additional file 3). BDX008 stratifies patients into two groups, BDX008+ and BDX008-, with better and worse outcomes on immunotherapy.

The test was applied without changes to the described cohort of advanced melanoma patients blinded to clinical data.

\section{Statistical analysis}

All analyses were performed using SAS9.3, SAS Enterprise Guide (SAS Institute, Cary, NC) or PRISM (GraphPad, La Jolla, CA).

Survival plots and medians were generated using the Kaplan-Meier method. Hazard ratios and $P$ values were calculated using Cox proportional hazard models; all $\mathrm{P}$ values are two-sided.

\section{Results}

Thirty patients (42\%) were classified as BDX008+ and 41 (58\%) were classified as BDX008-. Patient characteristics by BDX008 classification for the whole cohort and for subgroups with and without $B R A F$ mutations are presented in Table 1. There was no significant correlation of BDX008 classification with BRAF status (Fisher's test
$P=0.605)$ and other clinical characteristics. However BDX008- classification was correlated with higher levels of lactate dehydrogenase ( $\mathrm{LDH})$, (Fisher's test $P=0.006$ at $\mathrm{LDH}$ cutoff of twice the upper limit of normal $\left(\mathrm{ULN}^{1}\right)$ ) and with NLR $\geq 5$ (Fisher's test $P=0.003$ ).

Median PFS and OS for the whole cohort were 3.2 months and 9.9 months, respectively. Unselected by BDX008, patients with $B R A F$ mutations had numerically shorter median PFS: 2.6 months vs 5.1 months and OS: 5.5 months vs 15.7 months, than patients with wild type $B R A F$. The differences, however, were not statistically significant: PFS HR = 1.21 (95\% CI: 0.70-2.09), $P=0.487$; OS HR $=1.37$ (95\% CI: 0.76-2.46), $P=0.291$. Of note is that patients with $B R A F$ mutations tended to be treated with the anti-PD-1 agent in higher lines (average/median number of lines $2.8 / 3$ in $B R A F$ WT and $3.7 / 4$ in $B R A F$ MUT patients, Fisher's test $P=0.076$ for patients in 2nd line vs. higher lines). Three of the BRAF WT patients had uveal melanoma; with these patients excluded BRAF WT patients had median PFS and OS of 8.0 months and 16.4 months, respectively. The difference in PFS and OS between non-uveal BRAF WT and BRAF MUT patients remained not statistically significant (data not shown).

BDX008+ classification was correlated with best overall response $(P=0.005)$, objective response rate $(P=0.056$, trend), and disease control rate $(P=0.002)$ (details in Additional file 1: Table S2). OS and PFS results stratified by BDX008 are summarized in Table 2 and Fig. 1 . In the whole cohort $(N=71)$, patients classified as BDX008+ had longer PFS and OS than BDX008- patients: median PFS 10.8 vs 2.8 months, $\mathrm{HR}=0.61$ (95\% CI: $0.37-1.02), P=$ 0.060 ; median OS 18.3 vs 4.9 months, $\mathrm{HR}=0.50$ (95\% CI: $0.29-0.88), P=0.016$. Similar results were observed in the $B R A F$ WT (not-uveal) $(N=36)$ subgroup for OS: BRAF WT BDX008+ patients had significantly better OS than $B R A F$ WT BDX008- patients (HR $=0.41$ (95\% CI: 0.180.93), $P=0.032$ ); median $\mathrm{OS}$ was 32.5 months and 6.0 months in BRAF WT BDX008+ and BDX008- patients, respectively. In PFS the separation was not statistically significant: median PFS was 18.7 months in BDX008+ vs 3.0 months in BDX008- patients ( $\mathrm{HR}=0.70$ 95\% CI: $0.35-$ 1.42), $P=0.321$ ) (Table $2 \mathrm{~B}$ ). Outcome differences in patients with BRAF MUT $(N=25)$ with respect to BDX008 classification were not statistically significant (PFS HR = 0.55 (95\% CI: 0.23-1.36), $P=0.196$; OS HR $=0.73$ (95\% CI 0.29-1.80), $\quad P=0.489)$, though numerically BDX008+ $B R A F$ MUT patients had better outcomes than BDX008$B R A F$ MUT patients: median PFS was 4.5 months vs 2.2 months, median OS was 12.3 months vs 2.9 months, respectively (Table $2 \mathrm{C}$ ). It appears that BRAF WT patients classified as BDX008+ have especially good outcomes on anti-PD-1 treatment, while patients classified as BDX008have poor prognosis on immunotherapy independently of their mutation status (Fig. 1, c-d). In the exploratory 
Table 1 Patient characteristics by BDX008 classification for all patients and by BRAF status

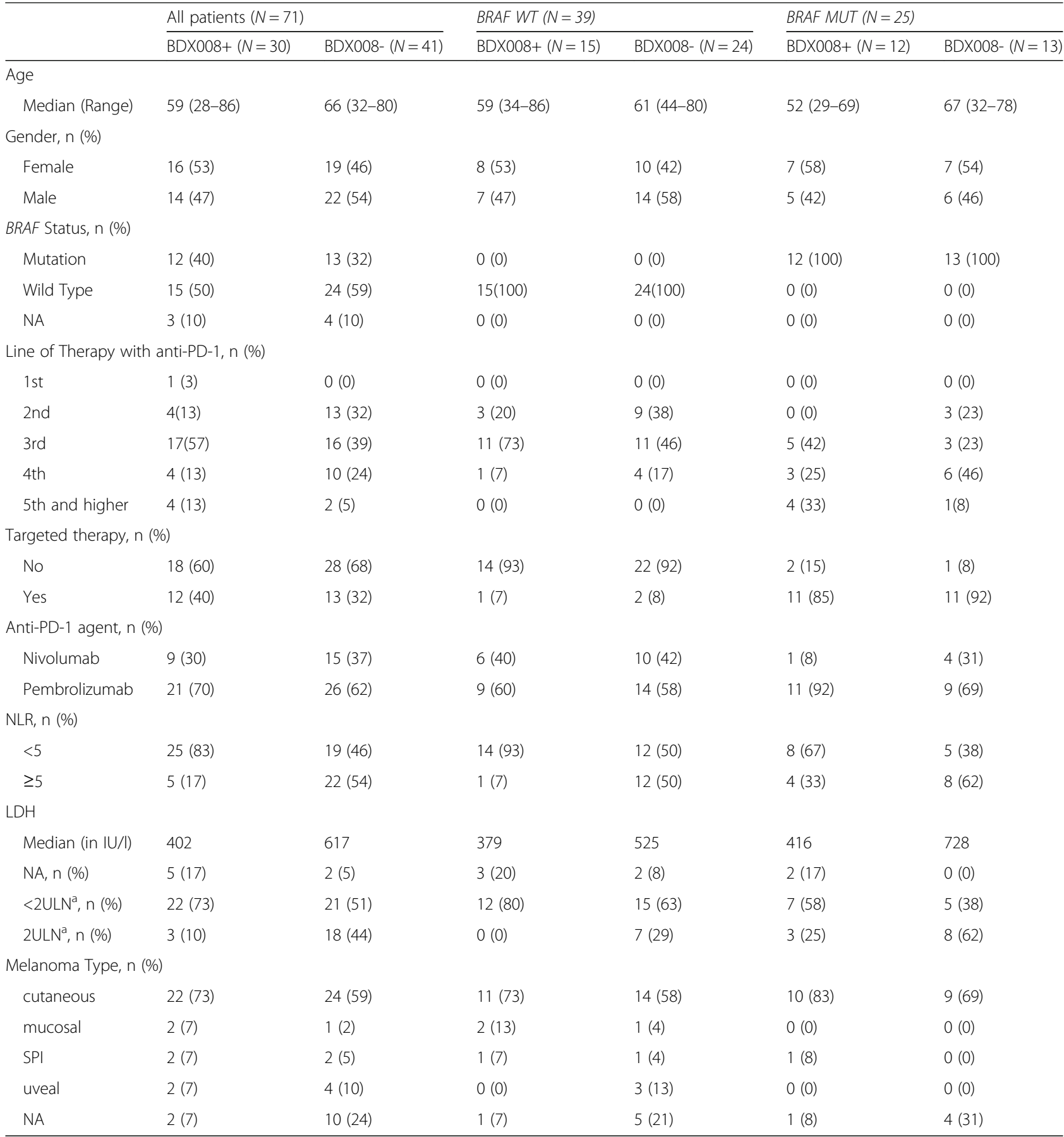

aLN upper limit of normal (333 IU/I)

analysis, when patients were stratified by NLR, BDX008+ classification was associated with improved OS in the low NLR subgroup $(N=44)$ : median OS was 29.7 months vs 6.8 months in BDX008+ and BDX008-, respectively, $\mathrm{HR}=0.38$ (95\%CI: 0.19-0.79, $P=0.008)$ (Table $2 \mathrm{D}$, Fig 1 e-f); BDX008+ patients also had numerically higher PFS (13.2 months vs 2.9 months in BDX008-, $P=0.169$ ).
In the high NLR subgroup ( $\mathrm{N}=25)$ BDX008 classification was not associated with PFS or OS (Table 2 E, Fig. 1 e-f).

In multivariate analyses adjusted for mutation status, line of treatment, and LDH level, BDX008 classification remained significantly associated with PFS and OS $(P=$ 0.031 and 0.009 , respectively); high LDH and higher line of treatment $(>2)$ were also significantly associated with worse 
Table 2 Treatment outcomes by test classification for the overall population (A), BRAF WT and BRAF MUT subgroups (B, C); and NLR $<5$ and $N L R \geq 5$ subgroups $(D, E)$

\begin{tabular}{|c|c|c|c|c|}
\hline \multirow[t]{2}{*}{ Classification } & \multicolumn{2}{|l|}{ PFS } & \multicolumn{2}{|l|}{ OS } \\
\hline & BDX008- & BDX008+ & BDX008- & BDX008+ \\
\hline \multicolumn{5}{|l|}{ A: All patients $(N=71)$} \\
\hline 2 years PFS or OS & $17 \%$ & $20 \%$ & $22 \%$ & $43 \%$ \\
\hline Median, months (95\% Cl) & $2.8(2.1-3.2)$ & $10.8(5.1-19.7)$ & $4.9(2.9-9.8)$ & $18.3(12.6-41.4)$ \\
\hline$H R-v s+(95 \% C l)$ & $0.61(0.37-1.02)$ & & $0.50(0.29-0.88)$ & \\
\hline$P$ value & 0.060 & & 0.016 & \\
\hline \multicolumn{5}{|c|}{ B: BRAF WT, excluding uveal $(\mathrm{N}=36)$} \\
\hline 2 years PFS or OS & $24 \%$ & $20 \%$ & $24 \%$ & $53 \%$ \\
\hline Median, months (95\% Cl) & $3.0(2.1-9.99)$ & $18.7(5.1-22.7)$ & $6.0(3.1-18.0)$ & $32.5(12.6-$ Undf*) \\
\hline$H R-v s+(95 \% C l)$ & $0.70(0.35-1.42)$ & & $0.41(0.18-0.93)$ & \\
\hline$P$ value & 0.321 & & 0.032 & \\
\hline \multicolumn{5}{|l|}{ C: BRAF MUT $(N=25)$} \\
\hline 2 years PFS or OS & $8 \%$ & $25 \%$ & $23 \%$ & $33 \%$ \\
\hline Median, months (95\% Cl) & $2.2(0.99-3.4)$ & 4.5 (0.99-Undf) & $2.9(2.1-11.2)$ & 12.3 (1.25-Undf) \\
\hline$H R-v s+(95 \% C l)$ & $0.55(0.23-1.36)$ & & $0.73(0.29-1.80)$ & \\
\hline$P$ value & 0.196 & & 0.489 & \\
\hline \multicolumn{5}{|l|}{$D: N L R<5(N=44)$} \\
\hline 2 years PFS or OS & $21 \%$ & $20 \%$ & $21 \%$ & $52 \%$ \\
\hline Median, months (95\% CI) & $2.9(2.2-10.0)$ & $13.2(6.2-22.7)$ & $6.8(2.9-15.7)$ & 29.7 (15.2-Undf) \\
\hline$H R-v s+(95 \% C l)$ & $0.63(0.33-1.22)$ & & $0.38(0.19-0.79)$ & \\
\hline$P$ value & 0.169 & & 0.008 & \\
\hline \multicolumn{5}{|l|}{$\mathrm{E}: \mathrm{NLR} \geq 5(\mathrm{~N}=27)$} \\
\hline 2 years PFS or OS & $14 \%$ & $0 \%$ & $23 \%$ & $0 \%$ \\
\hline Median, months (95\% Cl) & $2.4(1.0-3.4)$ & $1.1(0.6-5.1)$ & $3.8(2.1-11.2)$ & $1.8(0.99-8.6)$ \\
\hline$H R-v s+(95 \% C l)$ & $1.88(0.69-5.14)$ & & $1.80(0.65-5.00)$ & \\
\hline$P$ value & 0.220 & & 0.255 & \\
\hline
\end{tabular}

*Undf $=$ Undefined

outcomes $(P=0.027$ and 0.011 for LDH and $P=0.016$ and 0.008 for line of treatment for PFS and OS, respectively), while $B R A F$ mutations were not significant $(P=0.895$ and 0.793 for PFS and OS, respectively), see Table 3, A. In the multivariate analysis of OS that included all these factors plus NLR as variables, line of treatment and LDH remained significant $(P=0.010$ and 0.008 , respectively), while BDX008 and NLR trended towards significance $(P=0.097$ and 0.094, respectively (Additional file 1: Table S3). The Kaplan-Meier plots (Fig. 1 e-f) suggested that the effect may be qualitatively different in the subgroups. Indeed, the analysis of interaction between BDX008 classification and NLR (Table 3 B) was significant both in PFS $(P=0.041)$ and OS $(P=$ $0.004)$, confirming the importance of both variables for prognosis.

\section{Discussion}

The goal of this retrospective study was to validate the previously developed BDX008 test in melanoma patients treated with anti-PD-1 therapy and to evaluate its role depending on BRAF mutation status. The results confirmed the prognostic property of the test in the whole cohort and in the BRAF WT patients, while in the BRAF MUT subgroup, numerical advantage in PFS and OS of patients with BDX008+ classification did not reach statistical significance. Importantly, multivariate analysis confirmed that BDX008 classification was significantly associated with PFS and OS independently of BRAF status, line of treatment, and LDH; LDH and treatment line were also significant prognostic factors in the analysis, while $B R A F$ status was not. Additional exploratory analysis evaluated the effect of the test in relation to NLR another prognostic factor associated with systemic inflammation. Correlation between high NLR and poor outcomes in melanoma and other solid tumors, including those treated with immunotherapies, was demonstrated previously $[20,21]$. We observed a significant interaction between the two factors in PFS and OS; it 
a
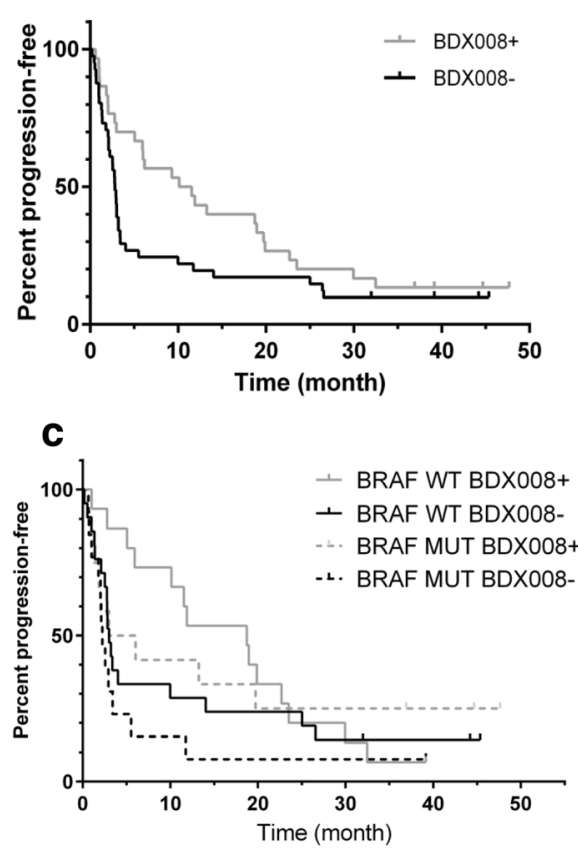

e

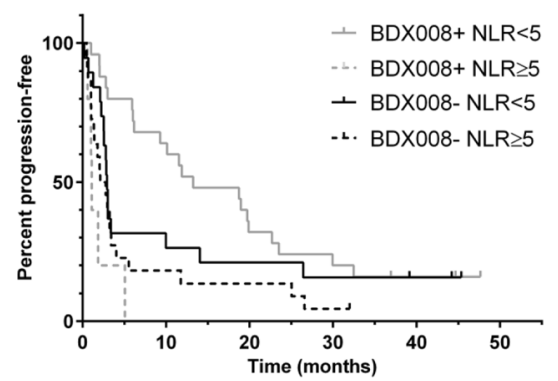

b

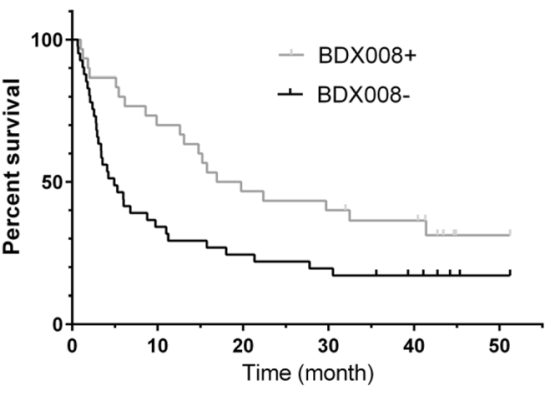

d

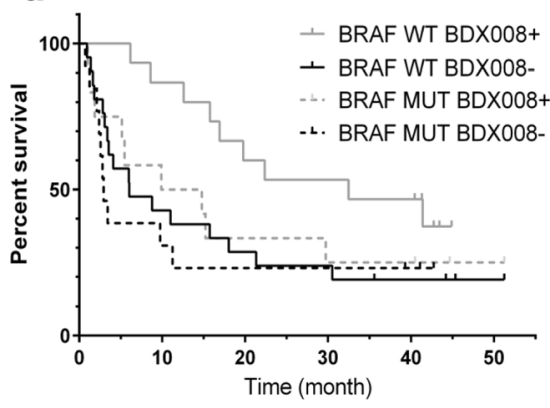

f

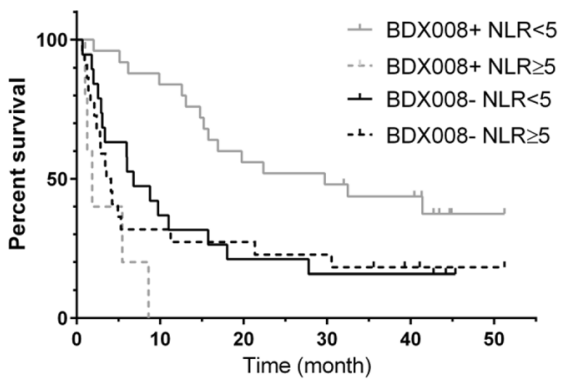

Fig. 1 Kaplan-Meier plots of outcome data by BDX008 classification for the whole cohort (a-b), for patients in subgroups with known BRAF status (c-d), and for patients in subgroups defined by NLR (e-f)

Table 3 Multivariate analysis of PFS and OS (A) - un-stratified, (B) - stratified by treatment line, including interaction of NLR and BDX008

\begin{tabular}{|c|c|c|c|c|}
\hline & \multicolumn{2}{|l|}{ PFS } & \multicolumn{2}{|l|}{ OS } \\
\hline & $P$ & $\mathrm{HR}(95 \% \mathrm{Cl})$ & $P$ & $\mathrm{HR}(95 \% \mathrm{Cl})$ \\
\hline \multicolumn{5}{|l|}{$A$} \\
\hline BDX008 (+ vs -) & 0.031 & $0.51(0.28-0.94)$ & 0.009 & $0.42(0.21-0.81)$ \\
\hline BRAF (MUT vs WT) & 0.895 & $0.96(0.50-1.85)$ & 0.793 & $1.09(0.56-2.14)$ \\
\hline $\operatorname{Line}^{a}$ (> 2 vs 2) & 0.016 & $2.35(1.17-4.71)$ & 0.008 & $3.01(1.33-6.82)$ \\
\hline LDH (high ${ }^{\mathrm{b}}$ vs low) & 0.027 & $2.26(1.10-4.67)$ & 0.011 & $2.60(1.24-5.43)$ \\
\hline LDH (n/a vs low $\left.{ }^{b}\right)$ & 0.322 & $1.53(0.66-3.56)$ & 0.332 & $1.56(0.63-3.86)$ \\
\hline \multicolumn{5}{|l|}{ B } \\
\hline BDX008 (+ vs -) & 0.096 & $0.57(0.29-1.11)$ & 0.002 & $0.32(0.15-0.66)$ \\
\hline$N L R \geq 5$ vs $<5$ & 0.183 & $1.57(0.81-3.03)$ & 0.592 & $1.20(0.61-2.37)$ \\
\hline BDX008*NLR interaction & 0.041 & $3.57(1.06-12.03)$ & 0.004 & $6.53(1.82-23.45)$ \\
\hline
\end{tabular}

a Line of anti-PD-1 therapy

${ }^{b}$ High LDH $>2$ ULN, Low LDH $<2$ ULN 
appears that in patients with BDX008+ classification NLR plays an important role, while in patients with poor prognosis by BDX008, PFS and OS are similar for high and low NLR patients (Fig. 1 e-f). Considering that patients with low NLR and BDX008+ had landmark 2 years survival of $52 \%$ and median OS of 28.7 months in advanced lines of treatment with immunotherapy, further studies of using these two biomarkers in combination are warranted.

Small sample size, especially in the BRAF MUT subgroup $(N=25)$ and $\mathrm{NLR} \geq 5(N=27)$, is a significant limitation of this study, resulting in reduced power of the statistical analysis and preliminary nature of the results. Another limitation is a difference in prior therapy and number of prior treatments between the subgroups, which allows only for qualitative comparisons. However, the results appear to be consistent and could be of clinical relevance. Overall, patients with $B R A F$ mutations had worse outcomes than BRAF WT patients, which could be due to a combination of several factors, such as more previous lines of therapy or poorer sensitivity to anti-PD-1 agents of these patients, when used after treatment with BRAF and MEK inhibitors. Additionally, prior targeted therapy may select for more aggressive disease which is harder to treat, possibly leading to worse outcomes. The multivariate analysis indicated that the line of treatment, rather than $B R A F$ mutation status, is significantly correlated with outcomes; however, the majority of patients with $B R A F$ mutations had, on average, more lines of treatment and $88 \%$ of them were treated with BRAF and /or MEK inhibitors in prior lines.

An important aspect of the BDX008 test is that, instead of focusing on few known molecular markers, BDX008 is a truly multivariate classifier, utilizing information pertinent to the circulating proteome in an unbiased, hypothesis-free way (for details, see Supplementary materials, Additional file 3), tending to reflect the systemic host response to the disease. Subsequent analysis of correlations between test classifications and various biological functions can provide insights into the mechanisms of sensitivity and resistance associated with the test. By applying a gene set enrichment analysis (GSEA) approach [22] to protein expression data, the BDX008 test was shown to be associated with acute phase reactants, wound healing, and complement activation [17]. Independent studies have demonstrated that complement activation can downregulate adaptive antitumor immunity [23], while chronic inflammation, characterized by pathological activation of wound healing processes and up-regulation of various acute phase reactants, creates a tumor-supportive and immune-suppressive microenvironment by activating MAPK pathways, affecting secretion of cytokines, and influencing innate and adaptive immune cells [24]. Observation of significant interaction between NLR and BDX008 in our study is intriguing, because it indicates that while both factors are related to systemic inflammation, they are not equivalent and capture different aspects of the state of the organism, which merits further exploration.

Up-regulation of inflammatory/acute response processes in treatment-naïve patients, as well as a result of prior therapies, including with BRAF/MEK inhibitors, may be part of the biological mechanism related to the poor prognosis associated with the BDX008- classification and with differences in the performance of the BDX008 test in population subgroups, which appears to work better in patients without BRAF mutations and in patients with low NLR. However, the effect of smaller sample size in the subgroups, resulting in diminished power of the analysis and, consequently, in the lack of significance in differences between BDX008- and BDX008+ in PFS and OS, also cannot be excluded. Hence, a larger study is needed to confirm the difference between the performance of the test in BRAF WT and $B R A F$ MUT populations, and to elucidate whether it is defined by the biological aspects related to $B R A F$ status or to prior therapy. Notably, patients classified as BDX008+ who were BRAF WT or had low NLR, demonstrated especially good outcomes, with median OS exceeding 32 months and 53\% landmark 2 years survival, highlighting the clinical utility of the test for predicting good prognosis on anti-PD-1 monotherapy in these groups of advanced melanoma patients.

\section{Conclusions}

In conclusion, this study independently validated previous results that BDX008 stratifies melanoma patients treated with anti-PD-1 agents into groups with better and worse PFS and OS in a mutation-unselected population and in $B R A F$ WT patients; its role in patients with $B R A F$ mutations and in relation to prior treatments needs to be confirmed in larger patient cohorts. In this study, treatment with anti-PD-1 of BRAF MUT patients who were classified as BDX008- resulted in numerically worse outcomes; BDX008+ patients had generally better prognosis, and BDX008+ BRAF WT patients had the best outcomes. In patients unselected by BRAF status, the best outcomes were observed in patients with low NLR and BDX008+ classification, indicating the possibility of further refinement of therapy using two biomarkers. However the small sample size and retrospective nature of the study requires further validation of these findings.

\section{Additional files}

Additional file 1: Additional tables. (DOCX $21 \mathrm{~kb}$ )

Additional file 2: Clinical information and outcomes. (XLSX 27 kb)

Additional file 3: Supplementary material. (DOC $84 \mathrm{~kb}$ ) 


\section{Abbreviations}

CTLA-4: Cytotoxic T-lymphocyte antigen-4; HR: hazard ratio; LDH: lactate dehydrogenase; MAPK: mitogen-activated protein kinase; NLR: neutrophil-tolymphocyte ratio; OS: overall survival; PD-1: programmed cell death protein 1; PD-L1: programmed cell death protein 1 ligand; PFS: progression-free survival; ULN: upper limit of normal

\section{Acknowledgements}

Not applicable.

\section{Funding}

This work was supported by grants from the Italian Ministry of Health (IT$\mathrm{MOH}$ ) through "Ricerca Corrente". MG receives funding from the institutional "Ricerca Corrente".

\section{Availability of data and materials}

The datasets supporting the conclusions of this article are included within the article (and its additional files).

\section{Authors' contributions}

$J G, H R, J R$ and PAA contributed to the study concepts and study design. Data acquisition was performed by GM, MC, DM, AMG, and ES; spectral acquisition, analysis, and test classification were performed by SA, CO and $\mathrm{KM}$. All the authors contributed to the quality control, analysis and interpretation of data. The statistical analysis was performed by JR and JG. Manuscript preparation was performed by JG and edited by HR and PAA. All authors reviewed and approved the manuscript.

\section{Ethics approval and consent to participate}

The study was approved by the Ethics Committee of the Istituto Nazionale Tumori IRCCS Fondazione "G. Pascale" with registration number 35/16 and all patients signed informed consent for the use of biological samples for research purposes

\section{Consent for publication}

Not applicable.

\section{Competing interests}

P.A: Consulting or Advisory Role for BMS, Roche-Genentech, MSD, Array, Novartis, Merck Serono, Pierre Fabre, Incyte, Genmab, Newlink Genetics, Medimmune, AstraZeneca, Syndax, Sun Pharma, Sanofi, Idera, Ultimovacs; Research funds from BMS, Roche-Genentech, Array; Travel support by MSD. A.G.: Consulting or Advisory Role for BMS, MSD, Novartis; Travel support by BMS, MSD, Merck Serono, Roche.

$H R, J R, J G, K M, S A, C O$ are or were full time employees of Biodesix and have/ had stock options of the company.

Other authors declare that they have no competing interests.

\section{Publisher's Note}

Springer Nature remains neutral with regard to jurisdictional claims in published maps and institutional affiliations.

\section{Author details}

"Istituto Nazionale Tumori IRCCS Fondazione "G. Pascale", Naples, Italy. ${ }^{2}$ Department of Translational Medical Sciences and Center for Basic and Clinical Immunology Research (CISI), University of Naples Federico II, 80131 Naples, Italy. ${ }^{3}$ Biodesix Inc, Boulder, CO, USA.

Received: 9 December 2018 Accepted: 15 March 2019

Published online: 29 March 2019

\section{References}

1. Chapman PB, Einhorn LH, Meyers ML, et al. Phase III multicenter randomized trial of the Dartmouth regimen versus dacarbazine in patients with metastatic melanoma. J Clin Oncol. 1999;17:2745-51.

2. Long GV, Weber JS, Infante JR, et al. Overall survival and durable responses in patients with BRAF V600-mutant metastatic melanoma receiving Dabrafenib combined with Trametinib. J Clin Oncol. 2016;34:871-8.

3. Dummer R, Ascierto PA, Gogas HJ, et al. Overall survival in patients with BRAF-mutant melanoma receiving encorafenib plus binimetinib versus vemurafenib or encorafenib (COLUMBUS): a multicentre, open-label, randomised, phase 3 trial. Lancet Oncol. 2018;19:1315-27.

4. Hodi FS, Chiarion-Sileni V, Gonzalez R, et al. Nivolumab plus ipilimumab or nivolumab alone versus ipilimumab alone in advanced melanoma (CheckMate 067): 4-year outcomes of a multicentre, randomised, phase 3 trial. Lancet Oncol. 2018.

5. Schachter J, Ribas A, Long GV, et al. Pembrolizumab versus ipilimumab for advanced melanoma: final overall survival results of a multicentre, randomised, open-label phase 3 study (KEYNOTE-006). Lancet. 2017;390: 1853-62.

6. Long GV, Schachter J, Ribas A, et al. 4-year survival and outcomes after cessation of pembrolizumab (pembro) after 2-years in patients (pts) with ipilimumab (ipi)naive advanced melanoma in KEYNOTE-006. Journal of Clinical Ocology. 2018;36.

7. Hodi FS, O'Day SJ, McDermott DF, et al. Improved survival with ipilimumab in patients with metastatic melanoma. N Engl J Med. 2010;363:711-23.

8. Schadendorf D, Hodi FS, Robert C, et al. Pooled analysis of Long-term survival data from phase II and phase III trials of Ipilimumab in Unresectable or metastatic melanoma. J Clin Oncol. 2015;33:1889-94.

9. Robert C, Long GV, Brady B, et al. Nivolumab in previously untreated melanoma without BRAF mutation. N Engl J Med. 2015;372:320-30.

10. Robert C, Schachter J, Long GV, et al. Pembrolizumab versus Ipilimumab in advanced melanoma. N Engl J Med. 2015;372:2521-32.

11. Dummer R, Ascierto PA, Gogas $H$, et al. Overall survival in COLUMBUS: A phase 3 trial of encorafenib (ENCO) plus binimetinib (BINI) vs vemurafenib (VEM) or enco in BRAF-mutant melanoma. In: 2018 ASCO Annual Meeting. Chicago, IL: J Clin Oncol; 2018:abst 9504.

12. Ascierto PA, Ferrucci PF, Stephens R, et al. KEYNOTE-022 part 3: phase II randomized study of $1 \mathrm{~L}$ dabrafenib (D) and trametinib (T) plus pembrolizumab (Pembro) or placebo (PBO) for BRAF-mutant advanced melanoma. Ann Oncol. 2018;29:mdy289-mdy.

13. Minor DR, Puzanov I, Callahan MK, Hug BA, Hoos A. Severe gastrointestinal toxicity with administration of trametinib in combination with dabrafenib and ipilimumab. Pigment cell \& melanoma research. 2015;28:611-2.

14. Hugo W, Zaretsky JM, Sun L, et al. Genomic and transcriptomic features of response to anti-PD-1 therapy in metastatic melanoma. Cell. 2016;165:35-44.

15. Hu-Lieskovan S, Robert L, Homet Moreno B, Ribas A. Combining targeted therapy with immunotherapy in BRAF-mutant melanoma: promise and challenges. J Clin Oncol. 2014;32:2248-54.

16. Weber JS, Kudchadkar RR, Yu B, et al. Safety, efficacy, and biomarkers of nivolumab with vaccine in ipilimumab-refractory or -naive melanoma. J Clin Oncol. 2013;31:4311-8.

17. Weber J, Martinez A, Roder H, et al. Pre-treatment patient selection for nivolumab benefit based on serum mass spectra. Journal for immunotherapy of cancer. 2015;3(Suppl 2):P103.

18. Grossi F, Rijavec E, Biello F, et al. Evaluation of a pretreatment serum tests for nivolumab benefit in patients with non-small cell lung cancer. J Thorac Oncol. 2017;12:S1322.

19. Zahorec R. Ratio of neutrophil to lymphocyte counts--rapid and simple parameter of systemic inflammation and stress in critically ill. Bratisl Lek Listy. 2001;102:5-14.

20. Capone M, Giannarelli D, Mallardo D, et al. Baseline neutrophil-to-lymphocyte ratio (NLR) and derived NLR could predict overall survival in patients with advanced melanoma treated with nivolumab. I Immunother Cancer. 2018;6:74.

21. Ferrucci PF, Ascierto PA, Pigozzo J, et al. Baseline neutrophils and derived neutrophil-to-lymphocyte ratio: prognostic relevance in metastatic melanoma patients receiving ipilimumab. Ann Oncol. 2018;29:524.

22. Subramanian A, Tamayo P, Mootha VK, et al. Gene set enrichment analysis: a knowledge-based approach for interpreting genome-wide expression profiles. Proc Natl Acad Sci U S A. 2005;102:15545-50.

23. Markiewski MM, DeAngelis RA, Benencia F, et al. Modulation of the antitumor immune response by complement. Nat Immunol. 2008;9:1225-35.

24. Crusz SM, Balkwill FR. Inflammation and cancer: advances and new agents. Nat Rev Clin Oncol. 2015;12:584-96. 